\title{
Piccolo Glossario per una migliore comunicazione tra MMG e specialista nefrologo
}

\author{
Sara Battistoni ${ }^{1}$, Elisa Ricci ${ }^{1}$, Rachele Brugnano ${ }^{1}$, Piera Bertoldi ${ }^{1}$, Maurizio Postorino ${ }^{2}$, \\ Giuseppe Quintaliani ${ }^{3}$
}

\author{
${ }^{1}$ S.C. Nefrologia e Dialisi, Azienda Ospedale Perugia, Perugia \\ ${ }^{2}$ Unità Operativa di Nefrologia, Dialisi e Trapianto Renale, Ospedali Riuniti, Reggio Calabria \\ ${ }^{3}$ Coordinatore Governo Clinico SIN, Ospedale S. Maria della Misericordia, Perugia
}

\begin{abstract}
A SHORT GLOSSARY FOR A BETTER COMMUNICATION BETWEEN GENERAL PRACTITIONERS AND NEPHROLOGISTS
Abstract. End stage renal disease (ESRD) is a chronic disease with a progressively increasing burden. To date, about 3-million people with ESRD in Italy can be classified with stage 3/5 K-DOQI, but less than $30 \%$ of patients with stage 4-5 refer to nephrology outpatient clinics.

Despite such evidence, the current knowledge on disease burden and, therefore, on the need to manage and possibly prevent complications is rather limited. General practitioners (GPs) and nephrologists often experience difficulties to communicate and cooperate concerning the appropriate duration of the nephropathic patients' follow-up. As a consequence, delayed diagnoses and increased risk of life-threatening complications are often observed. Indeed, since ESRD is also a marker of concurrent cardiovascular disease, renal function or urinalysis abnormalities could suggest underlying cardiac diseases including ischemic cardiac disease.

Therefore, we created a glossary for GPs that includes information on both the diagnostic and the follow-up approach for nephropathic patients. We believe that this tool should allow identifying early metabolic abnormalities and optimizing the duration and management of the longitudinal follow-up.
\end{abstract}

Key words: CKD, Nephropatic patients, Follow-up, Metabolic complications

Conflict of interest: None.

Financial support: None.

Accettato: 29 Gennaio 2014

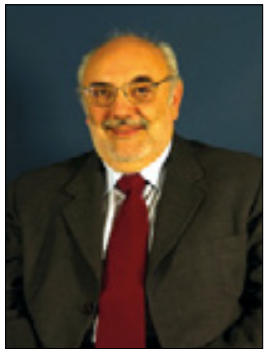

L'insufficienza renale cronica (IRC), definita come riduzione del GFR $<60 \mathrm{~mL} /$ $\mathrm{min} / 1.73 \mathrm{~m}^{2}$, è una delle patologie croniche in forte espansione, soprattutto per l'aumento dell'età media della popolazione e dell'incidenza di patologie cardiovascolari e del diabete.

La prevalenza della malattia renale cronica è in continuo aumento, anche se di difficile stima, e a ciò concorrono la Giuseppe Quintaliani scarsa sintomaticità e la bassa identificazione negli più precoci.

Facendo riferimento alla classificazione della malattia renale proposta dall'American Kidney Foundation e applicabile su scala mondiale (Fig. 1, classificazione K-DOQI), si stima che attualmente, in Italia, circa 3 milioni di pazienti con IRC siano agli stadi 3-5 sec. K-DOQI, ma una percentuale inferiore al 30\% di quelli agli stadi 4-5 sarebbe nota presso gli ambulatori nefrologici.
I soggetti con diabete, ipertensione arteriosa, malattie cardiache, obesità, grande anzianità, esposizione a fumo di sigaretta e basso stato sociale hanno maggiori probabilità di essere affetti da IRC. Tali fattori di rischio per la comparsa o la progressione dell'IRC sono in gran parte comuni a quelli per le malattie cardiovascolari. L'IRC è un marcatore di malattia cardiovascolare; alterazioni della funzione renale o dell'esame delle urine possono precedere la comparsa di sintomi di cardiopatia ischemica o l'evidenza strumentale o laboratoristica di una malattia cardiovascolare.

Un intervento precoce centrato sui pazienti con IRC aumenta le possibilità di successo della prevenzione e della cura delle malattie cardiovascolari; per tale motivo, la valutazione della funzione renale sta assumendo una crescente importanza, soprattutto in termini di prevenzione secondaria delle complicanze dell'insufficienza renale cronica, potenzialmente letali.

Purtroppo risulta, però, scarsa la consapevolezza delle dimensioni del problema e, di conseguenza, dell'importan- 


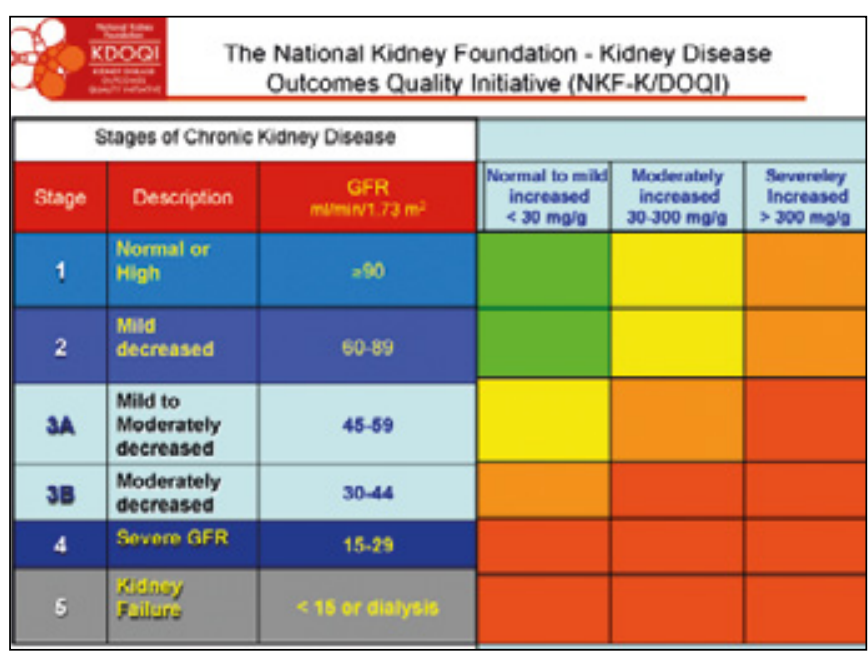

Fig. 1 - Classificazione K-DOQI.

za di un attento follow-up di questi pazienti, soprattutto per quanto riguarda la gestione e la prevenzione delle complicanze.

Le complicanze dovute all'IRC sono numerose e iniziano in una fase relativamente precoce della malattia (Fig. 2). La prevalenza crescente dell'IRC porta a una stretta sorveglianza di tale popolazione, condivisa tra Medico di Medicina Generale (MMG) e specialista: se la prevalenza della popolazione affetta da una riduzione della funzione renale fosse di circa 4 milioni ( $6 \%$ della popolazione), le strutture nefrologiche ben difficilmente potrebbero farsi carico dell'assessment e del follow-up di tale mole di pazienti.

Spesso, MMG e Specialisti si trovano in difficoltà a comunicare circa le modalità e i contenuti dei controlli longitudinali dei nefropatici inquadrati in schemi spesso poco chiari o poco condivisi.

Abbiamo ritenuto, quindi, utile fornire un piccolo Glossario che sottintende la valutazione diagnostica di base e il followup del nefropatico, con la duplice finalità di permettere una precoce identificazione delle alterazioni metaboliche e/o un controllo longitudinale scevro da accertamenti inutili o posticipazioni di visite per mancanza o insufficienza di dati. Questa review non vuole essere un percorso diagnosticoterapeutico delle nefropatie, ma un piccolo Glossario degli esami normalmente richiesti per il follow-up di una malattia renale già accertata.

La frequenza media della visita specialistica e degli esami di laboratorio dovrebbe essere di 6-9 mesi in classe DOQI 3 mentre, nel paziente con un'IRC classe DOQI 4-5 (GFR $<15$ $\mathrm{mL} / \mathrm{min}$ ), la distanza tra un controllo e l'altro può arrivare fino a 1 mese e mezzo circa.

\section{Prevalenza delle alterazioni tipiche della IRC per vari intervalli di funzione}

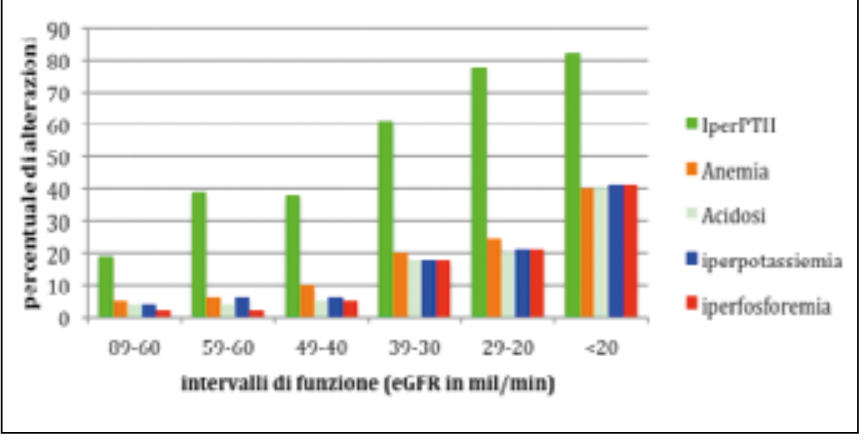

Fig. 2 - Le alterazioni connesse con la funzione renale nei vari stadi della malattia.

\section{Il Glossario dello screening nefrologico}

\section{AZOTEMIA}

Rappresenta il parametro di laboratorio con il quale si stima l'UREA, sostanza che si forma nel fegato alla fine dei processi di catabolismo delle proteine; in pratica, gli aminoacidi che non vengono utilizzati per scopi anabolici (formazione di nuove proteine) vengono immessi nel ciclo di Krebs e degradati nel fegato con formazione finale di urea, poi eliminata dal rene (1-6).

L'aumento di tale valore si verifica quando la clearance della creatinina, espressione del filtrato glomerulare, scende sotto i $50-60 \mathrm{~mL} / \mathrm{min}$ oppure in seguito a ipercatabolismo o, viceversa, per eccessiva introduzione di elementi proteici. Questo parametro è, quindi, indicativo di come e di quanto il paziente si alimenta. Un'azotemia aumentata può, infatti, essere riscontrata non solo per un'abbondante introduzione di proteine, ma anche in seguito a uno scarso introito di calorie e al conseguente dimagrimento: tale situazione prende il nome di gluconeogenesi.

La gluconeogenesi è quel processo metabolico che si attiva nel nostro organismo in risposta a una carenza di glucosio nel sangue. Tale zucchero viene, quindi, sintetizzato a partire da precursori di origine non glucidica e i substrati che vengono utilizzati per essere convertiti a glucosio sono specifici aminoacidi, glicerolo, acido lattico (lattato) e acido piruvico (piruvato). Dal catabolismo degli aminoacidi si forma poi l'urea, che viene metabolizzata ad azoto mediante il ciclo dell'urea (7). La gluconeogenesi, che è principalmente di competenza epati$c a$, anche se avviene in misura minore nei reni e nell'intestino, diventa fondamentale durante il digiuno o quando l'assunzione di calorie con la dieta è deficitaria, cioè quando le riserve glucidiche dell'organismo sono esaurite. Il cervello consuma circa $120 \mathrm{~g}$ di glucosio al giorno, mentre il fabbisogno quoti- 
diano dell'intero organismo ammonta a circa $200 \mathrm{~g}$, quindi, per garantire la continuità di approvvigionamento di glucosio ai tessuti che ne hanno bisogno, il corpo umano ha adottato tale strategia che converte le molecole meno mobili in glucosio (8). Talvolta, viene suggerita erroneamente al paziente nefropatico una dieta non solo ipoproteica, ma anche ipocalorica. La conseguenza che deriva dalla scarsa assunzione di calorie è, quindi, l'ipercatabolismo azotato, cioè l'aumento dell'azotemia in risposta alla necessità del nostro organismo di produrre energia.

Altro motivo frequente di iperazotemia è la disidratazione spesso indotta da diuretici, un meccanismo tipico dell'IRC pre-renale (per esempio, il paziente con scompenso cardiaco e importante terapia diuretica).

Una perfusione renale compromessa con una conseguente caduta della pressione di filtrazione glomerulare è una causa comune di IRA. In questa circostanza, si riscontrano un aumento del riassorbimento di acqua e sodio e, conseguentemente, un'urina più concentrata (osmolarità urinaria $>500 \mathrm{mOsm} /$ $\mathrm{Kg}$ ) e una riduzione della sodiuria $(<10 \mathrm{mmol} / \mathrm{L})(9)$.

Gli stadi di ipoperfusione renale inducono delle alterazione fisiopatologiche che sono finalizzate alla conservazione di acqua e di sodio e al ripristino di un volume circolante efficace. Aumentano, pertanto, il riassorbimento di acqua e sodio $\mathrm{e}$, in maniera passiva, quello dell'urea. Un ruolo importante in questi meccanismi adattativi è giocato dall' $\mathrm{ADH}$, rilasciato su base non osmotica dall'ipofisi, che media l'aumentato riassorbimento di acqua e urea dal dotto collettore per aumentata espressione del trasporto di questa molecola (10).

Circa il 40\% dell'osmolarità dell'interstizio della midollare è fornito dall'urea, essendo i dotti collettori di questa zona particolarmente permeabili a tale catabolita. L'urea contribuisce, infatti, non solo all'aumento dell'osmolarità dell'interstizio della midollare, ma anche alla formazione di urine concentrate. È per tale ragione che, in condizioni patologiche, di ipoperfusione, dove i meccanismi di autoregolazione non sono più efficaci, grandi quantità di urea sono riassorbite passivamente dai dotti collettori della midollare, favorendo, così, un'iperosmolarità dell'interstizio, un aumento dell'urea nel sangue e la formazione di urina più concentrata (11), cioè le caratteristiche che si riscontrano nel paziente disidratato con IRA.

\section{CREATINEMIA}

È una sostanza che proviene dal metabolismo della creatina, prodotta dai muscoli e, quindi, in stretta relazione con la massa muscolare. In generale, non è in relazione con l'alimentazione, non è riassorbita ed è solo in modestissima misura secreta dai vari segmenti del nefrone; è di facile determinazione laboratoristica. Per tali motivi è molto più affidabile dell'azotemia come marcatore della funzione renale. I kit per il dosaggio della creatinina in commercio sono numerosi e, purtroppo, molti di essi sono calibrati in modo differente, in modo tale che, in diversi laboratori, la misurazione di questo parametro può dare valori differenti, soprattutto quando è usata per la stima della funzione renale.

Per tale motivo, molti pazienti con creatinina definita normale dai laboratori hanno, invece, delle forti riduzioni del filtrato (Fig. 3).

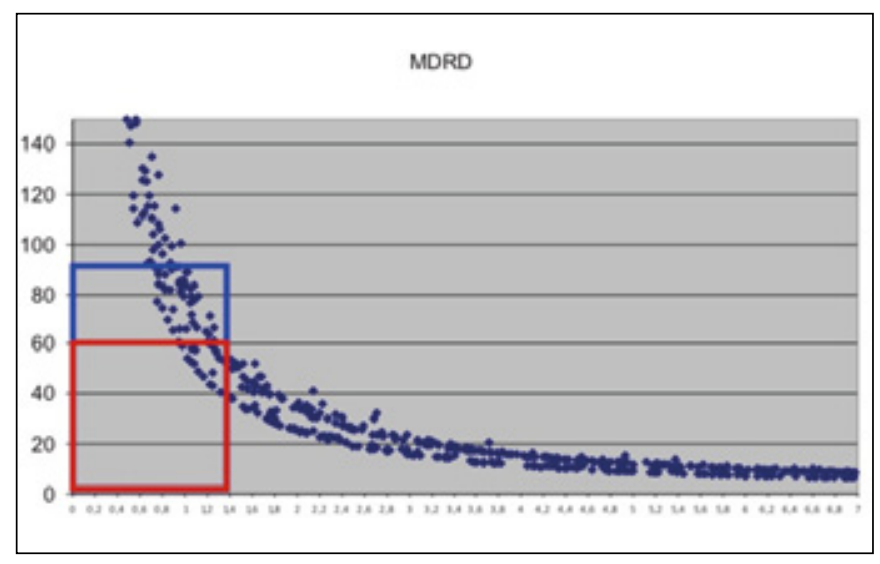

Fig. 3 - Plot della creatinina vs filtrato glomerulare stimato. Si nota che molte creatinine entro il range normale riflettono una riduzione della funzione renale anche molto importante.

\section{VALUTAZIONE DELLA FUNZIONE RENALE}

Proprio per l'inaffidabilità della sola creatinina è necessario ricorrere ad altri sistemi per la misura della funzione renale. La clearance creatinina è il più antico metodo di stima del volume di filtrato glomerulare (VFG), espresso come $\mathrm{mL}$ di sangue depurati nel tempo (un minuto) della creatinina. Ciò comporta la misura della creatinuria dosata sulla raccolta urinaria delle 24 ore. I valori di clearance della creatinina che provengono dal laboratorio sono riferiti alla superficie corporea dell'individuo e, in individui che si discostano molto dalla superficie corporea media, essi dovrebbero essere normalizzati per la superficie corporea standard $(1.73 \mathrm{mq})$. La clearance della creatinina sovrastima il VFG, soprattutto in corso di IRC, in quanto la creatinina viene secreta maggiormente a livello tubulare e la sovrastima è tanto maggiore quanto più la creatinina è elevata.

\section{Le formule per il calcolo della funzione renale}

Consentono di estrapolare il VFG sulla base della creatininemia e di altre variabili antropometriche e demografiche che, non richiedendo la raccolta urinaria, risultano, così, di più facile applicazione.

La formula più utilizzata per il calcolo della funzione renale è quella derivata dallo studio MDRD (Modification Diet in Renal Disease) (12), che stima il filtrato glomerulare sulla base di creatinina, età e sesso del paziente, con il limite di una possibile sottostima del VFG, fino al $15 \%$, nei soggetti con funzione renale normale (12-14). Inoltre, tale formula non è stata validata in pazienti oltre il $75^{\circ}$ anno di vita; lo scarto potrebbe aggirarsi intorno ai $5-7 \mathrm{~mL} / \mathrm{min}$ e, quindi, la formula risulta utilizzabile per motivi clinici mentre, per screening e studi, potrebbe comportare qualche bias. Dal momento che non sono necessari altri parametri clinici, è spesso riportata in automatico da molti laboratori analisi. Un'altra formula molto utilizzata per la stima della funzione renale è la CockroftGault, che stima la clearance della creatinina e non il filtrato. Richiede, però, per la sua determinazione, il peso corporeo. 
In caso di sovrappeso dovuto a obesità, potrebbe essere necessario inserire non il peso attuale ma quello desiderabile, per evitare problemi di sovrastima (in soggetti con BMI $>30$ ). Per superare il problema dell'erronea classificazione dei pazienti nefropatici per sovra/sottostima della clearance, è stata recentemente studiata una nuova formula, che corregge parzialmente l'errore, ovvero la CKD-EPI (Chronic Kidney Disease Epidemiology Collaboration) (12), che si avvale necessariamente della creatinina calibrata (come, per esempio, quella utilizzata presso l'Azienda Ospedale di Perugia). L'obiettivo è di fornire una valutazione del VFG più accurata rispetto alle precedenti nei soggetti con funzione renale normale o lievemente ridotta (tra 90 e $60 \mathrm{~mL} / \mathrm{min}$ ) e ha dimostrato di aver raggiunto tale scopo fornendo, in questi soggetti, un valore più vicino a quello del VFG rispetto al MDRD.

La stadiazione dell'IRC secondo la K-DOQI utilizza la funzione renale per la suddivisione dei 5 stadi di insufficienza renale e per il monitoraggio longitudinale del paziente (Fig. 1). In rete esistono molti calcolatori nefrologici che possono dare risultati affidabili e attendibili $(13,14)$.

\section{EMOCROMO E ASSETTO MARZIALE}

Una delle complicanze più comuni della CKD è l'anemia, di tipo normocromico-normocitico, definita in base alla presenza di livelli di emoglobina $<11.5 \mathrm{~g} / \mathrm{dL}$ nella donna, $<13.5 \mathrm{~g} / \mathrm{dL}$ nell'uomo di età inferiore ai 70 anni e $<12$ g/dL nell'uomo di età pari o superiore ai 70 anni (European Best Practice Guidelines). La patogenesi dell'anemia in corso di IRC è correlata all'insufficiente produzione di eritropoietina endogena (EPO) da parte del parenchima renale e alla riduzione della vita media eritrocitaria per la presenza di tossine uremiche circolanti. Tuttavia, altre cause possono essere fattori determinanti, come limitata disponibilità di ferro per l'eritropoiesi, ridotta espressione dei recettori per l'EPO, infezioni e una serie di disfunzioni associate alla IRC, come iperparatiroidismo e carenza di folati e vitamina B12.

L'anemia può svilupparsi prima dell'insorgenza dei sintomi dell'uremia; benché l'anemia secondaria alla nefropatia generalmente si sviluppi quando il GFR si riduce a meno di 30 $\mathrm{mL} / \mathrm{min}$, un decremento dei livelli di emoglobina può essere osservato anche in soggetti con GFR più elevato $(45 \mathrm{~mL} / \mathrm{min})$, soprattutto nei soggetti diabetici. Oltre all'anemia, gli esami di laboratorio evidenziano una conta reticolocitaria ridotta, con una carenza marziale conseguente alle perdite croniche attraverso il tratto gastroenterico e durante la seduta dialitica. L'associazione con uno stato flogistico cronico può modificare l'assetto marziale, determinando quadri sovrapponibili all'anemia delle malattie croniche.

L'avvento della terapia con ESA (Erythropoiesis Stimulating Agents) ha determinato, di fatto, la completa correzione dell'anemia da IRC, l'eliminazione della dipendenza da trasfusioni periodiche e una riduzione della somministrazione di ferro nei pazienti con sovraccarico marziale (10). In questi pazienti non si raccomanda di eseguire il dosaggio di EPO, poiché non fornisce un parametro aggiuntivo nella guida della terapia con EPO.

La terapia sostitutiva con eritropoietina è indicata in quei pazienti con $\mathrm{Hb}<11 \mathrm{~g} / \mathrm{dL}$, previa ricostituzione dei depositi mar-

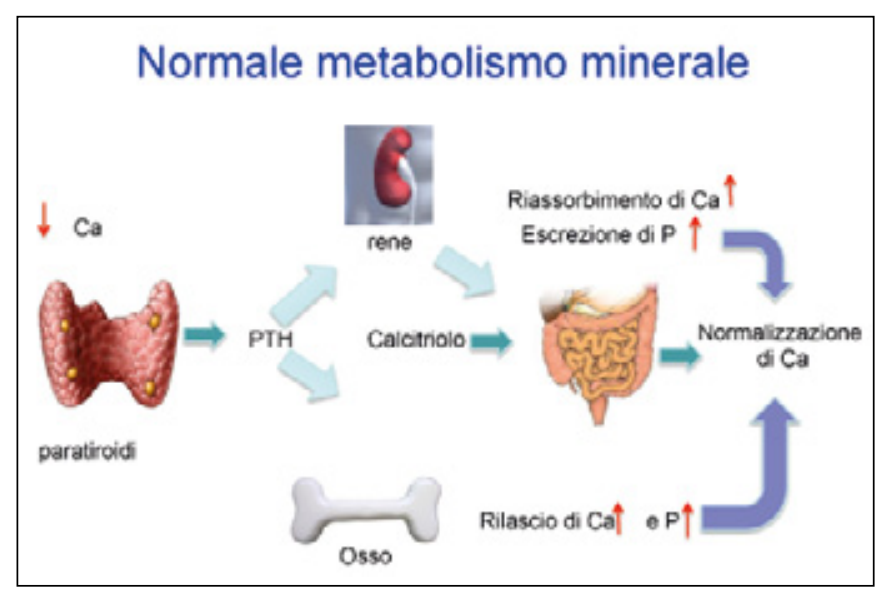

Fig. 4 - Metabolismo calcio-fosforo nell'IRC.

ziali (3); questo è un concetto fondamentale, poiché la terapia con EPO potrebbe non avere alcun effetto o, comunque, potrebbe avere un effetto modesto se manca il substrato del ferro. La terapia con ESA non va iniziata senza una contemporanea terapia marziale in presenza di carenza marziale assoluta (ferritinemia $<100 \mathrm{ng} / \mathrm{mL}$ ) o funzionale (TSAT $<20 \%$ e ferritinemia $>100 \mathrm{ng} / \mathrm{mL}$ ). La TSAT (saturazione della transferrina) rappresenta il rapporto tra il valore della sideremia (quantità di ferro di trasporto presente nel sangue - v.n. 60-175 mcg/dL) e della TIBC (Total Iron Binding Capacity), capacità totale di legare il ferro, in percentuale. Questo rapporto, (sideremia/TIBC) x 100, definisce la saturazione della transferrina, con valori normali nell'uomo del $20-50 \%$ e, nella donna, del $15-50 \%$.

Nei pazienti con IRC in terapia con ESA, i supplementi di ferro dovrebbero essere adattati per mantenere una TSAT $>20 \%$ e una ferritinemia tra 200 e $800 \mathrm{ng} / \mathrm{mL}$; per non superare gli $800 \mathrm{ng} / \mathrm{mL}$, il dosaggio del ferro dovrebbe essere riconsiderato quando la ferritinemia è $>500 \mathrm{ng} / \mathrm{mL}$ (SIN-ISS 2012, in accordo con KDIGO 2012) (Fig. 4).

Viene, inoltre, suggerito l'avvio con terapia orale, se tollerata, e controllando la sua efficacia, verificando che non vi sia l'assunzione contemporanea di farmaci che possano ridurne l'assorbimento: inibitori della pompa protonica, antiacidi e resine per controllare potassiemia e fosforemia.

Nel follow-up dell'anemia nell'IRC, il dato più importante da esaminare è la risposta reticolocitaria, che, in questi pazienti, risulta sotto i limiti della norma per il ridotto stimolo midollare da parte dell'EPO.

\section{METABOLISMO CALCIO-FOSFORO E PTH (CKD-MBD)} Il calcio e il fosforo sono i sali minerali presenti in maggiori quantità nel nostro organismo e hanno un importante ruolo nella produzione di energia, nello sviluppo osseo e cellulare e nella contrazione muscolare. Il metabolismo calcio-fosforo è un importante indice diagnostico e prognostico nell'IRC, direttamente collegato al paratormone (PTH). Solitamente, nell'insufficienza renale, si hanno IPERFOSFOREMIA, con- 
seguente al danno renale, agli introiti con la dieta e alla diminuita escrezione del fosforo, e IPOCALCEMIA, per mancata attivazione della vitamina $\mathrm{D}$ (Fig. 4).

L'IPERPARATIROIDISMO SECONDARIO che si sviluppa in risposta all'ipocalcemia e all'iperfosforemia (il PTH è in grado di mobilizzare il Ca dalle ossa e di aumentare l'escrezione urinaria di P) è una delle principali complicanze dell'IRC. Un aumento dei valori del PTH, spesso molto precoce, può portare, nel corso del tempo, a gravi danni renali, cardiaci e scheletrici.

I disturbi del metabolismo calcio-fosforo sono, infatti, alla base di malattie dell'osso (OSTEODISTROFIA RENALE) e delle calcificazioni dei tessuti molli e, soprattutto, vascolari, con un aumento della pressione arteriosa e dell'aterosclerosi. Questi disturbi rappresentano la prima causa di morte nel paziente in dialisi, pertanto la loro prevenzione e la loro terapia rappresentano un passaggio chiave nel trattamento del paziente con IRC agli stadi 3-5.

L'iperparatiroidismo, l'iperfosfatemia e il deficit di attivazione della vitamina $D$ hanno acquisito, pertanto, un valore clinico rilevante e nuovo, tanto che è stata recentemente definita una nuova entità nosologica, la malattia renale cronica con disordini del metabolismo minerale e dell'osso (CKD-MBD), che considera in modo unitario le alterazioni metaboliche, ossee e cardiovascolari del paziente con IRC $(15,16)$.

L'arteriolosclerosi calcifica e l'ipertrofia miocardica sono le alterazioni cardiovascolari più diffuse in questi pazienti e più predittive di mortalità e morbidità (17-19). L'arteriolosclerosi calcifica è il risultato della deposizione di fibre collagene e di idrossiapatite nella tonaca media delle arterie, con sviluppo di calcificazioni sub-continue della parete arteriosa e che si differenziano da quelle aterosclerotiche, irregolari e situate nell'intima (20).

L'arteriolosclerosi calcifica ha importanti conseguenze ischemiche locali ed emodinamiche, perché la rigidità vascolare che ne deriva determina un incremento della pressione sistolica con stimolo dell'ipertrofia del miocardio e anomala caduta pressoria durante la diastole, con compromissione del flusso ematico periferico $(21,22)$. In particolare, alcuni studi epidemiologici hanno evidenziato come l'iperparatiroidismo sia associato alla mortalità dei pazienti con diversi stadi di IRC (23$26)$ e in pazienti con iperparatiroidismo primitivo $(27,28)$.

In questi ultimi, è stato descritto un ispessimento dell'intima e della media senza la marcata tendenza alla calcificazione tipica della CKD ed è stata, inoltre, registrata la regressione delle alterazioni cardiache dopo la paratiroidectomia.

Il PTH, può, pertanto, innescare il processo di calcificazione arteriosa indipendentemente dalla presenza di iperfosforemia (29). Mentre questi risultati hanno evidenziato i risvolti negativi sul deterioramento arterioso secondario all'iperparatiroidismo, altri studi in diversi modelli animali hanno osservato un effetto benefico dello stesso, sulla funzione miocardica, sul tono arterioso, sulla contrattilità cardiaca e sulla vasodilatazione (30-32). Sebbene, comunque, questi effetti possano essere considerati funzionalmente positivi, il senso clinico che il PTH ha acquisito nella pratica clinica è, generalmente, negativo, perché la condizione di iperparatiroidismo, sia primitivo che secondario, è stata associata all'insorgenza di arteriosclerosi $(33,34)$ e ipertrofia cardiaca, con le relative complicanze (35-40).

Alcuni studi epidemiologici e sperimentali hanno identificato anche nell'IPERFOSFOREMIA un'alterazione chiave per lo sviluppo di CKD-MBD. L'incremento della fosforemia, sin da valori nel range di norma, è strettamente associato alla mortalità cardiovascolare nei pazienti in fase sia dialitica che predialitica $(41,42)$. Il meccanismo patogenetico è rappresentato dall'incremento dell'uptake del fosfato da parte delle cellule muscolari lisce e dall'innesco della differenziazione in senso osteoblastico delle cellule muscolari vascolari, che diventano capaci di depositare idrossiapatite e collagene tipo I, attivando il processo di calcificazione arteriosa (43).

In conclusione, gli studi finora condotti ci permettono di individuare nell'iperparatiroidismo un fattore prognostico negativo per i pazienti con IRC. L'iperparatiroidismo è diventato, così, un target di riferimento per la terapia medica conservativa della CKD-MBD, insieme all'iperfosforemia; non sono ancora chiari, purtroppo, i livelli plasmatici target, che verosimilmente cambiano a seconda dello stadio della IRC e in funzione di calcio-fosforo e vitamina D.

Il PTH andrebbe prelevato ogni 6 mesi circa; un aumento dei suoi valori può essere riscontrato agli esami laboratoristici precocemente dopo l'insorgenza dell'insufficienza renale e con filtrati appena sotto i $60 \mathrm{mil} / \mathrm{min}$.

\section{SODIO-POTASSIO}

Sono i cationi più importanti del nostro organismo. Essendo il sodio distribuito prevalentemente nel compartimento extracellulare, alterazioni dei suoi valori sono generalmente legate alle condizioni di idratazione del paziente, mentre il potassio risente molto meno dello stato di idratazione, essendo presente prevalentemente a livello intracellulare.

Nell'IRC raramente si assiste ad alterazioni del sodio e del potassio finché la funzione renale non si riduce progressivamente, cioè quando esiste una diuresi residua valida.

$\mathrm{Si}$ ha iperpotassiemia soprattutto nelle fasi avanzate della malattia. Talvolta, l'aumento della potassiemia può essere talmente lento e graduale da non manifestarsi clinicamente. Capita spesso di osservare un paziente con $7 \mathrm{mEq} / \mathrm{L}$ di potassio senza alcun sintomo cardiologico, stanchezza o crampi muscolari.

Altra causa importante di iperpotassiemia è l'utilizzo di alcuni farmaci anti-ipertensivi. In caso di iperpotassiemia persistente, è necessario controllare se il paziente assume una terapia con ACE-INIBITORI, SARTANICI e DIURETICI RISPARMIATORI DI POTASSIO!

Fondamentale in questi pazienti è il controllo dietetico; ridurre l'assunzione di sale e di cibi ricchi di potassio (banane, frutta fresca) permetterà di tenere sotto controllo i valori e di evitare gravi complicanze.

Attenzione, inoltre, a:

febbre o stati infettivi, in seguito ai quali possono svilupparsi marcata acidosi e peggioramento dell'iperpotassiemia improvvisa contrazione della diuresi

Nell'IRC con ipertensione è indicata anche la riduzione dell'introito del sodio che, però, non deve essere drastica. 
È sempre utile sorvegliare la sodiemia e la sodiuria per non correre il rischio di ridurre le riserve di sodio che, lo ricordiamo, non è obbligatoriamente perso nell'IRC, fatta eccezione per alcune patologie glomerulari.

\section{EMOGASANALISI}

Nell'IRC viene meno una delle funzioni principali del rene, che è quella di mantenere un adeguato equilibrio acido-base tramite riassorbimento tubulare e formazione dei bicarbonati. In genere, l'equilibrio si mantiene fino a quando il volume del filtrato glomerulare non scende al di sotto del $50 \%$ dei valori normali.

L'acidosi metabolica è una complicanza frequente nel paziente con insufficienza renale cronica. Nella maggior parte dei casi, è sufficiente un EGA venoso, dato che il principale parametro da valutare è la bicarbonatemia (HCO3-), per risparmiare sia discomfort al paziente che, soprattutto, il patrimonio vascolare, in vista di un potenziale allestimento di fistola AV per dialisi. La concentrazione dei bicarbonati può non raggiungere livelli estremamente bassi, grazie all'attivazione di meccanismi di compenso polmonare con ipocapnia. Nei casi più gravi, può manifestarsi dispnea, espressione del tentativo di compenso polmonare. Per ottenere un discreto compenso metabolico, i valori di HCO3- dovrebbero essere $>18-20 \mathrm{mmol} / \mathrm{L}$ (44).

Attenzione particolare meritano i soggetti diabetici in terapia con metformina, poiché, nei casi di insufficienza renale acuta di qualsiasi natura, su una base di cronicità, sono quelli più a rischio di acidosi severe (45). In ogni caso, la metformina andrebbe sospesa nei pazienti con filtrato inferiore a $40 \mathrm{~mL} / \mathrm{min}$.

\section{COLESTEROLO}

Il dosaggio dell'assetto lipidico viene considerato un indice importante di rischio cardiovascolare, che contribuisce indirettamente a una più rapida progressione del danno renale. Il controllo del colesterolo può essere effettuato non solo con una dieta adeguata, ma anche con l'aiuto di farmaci come le statine.

Inoltre, le Linee Guida sulla terapia dell'IRC (K-DIGO) mettono in risalto l'importanza dell'utilizzo delle statine nel trattamento dei pazienti nefropatici con ipercolesterolemia, in quanto sono in grado di ridurre il rischio cardiovascolare (46).

\section{$\underline{\text { AZOTURIA }}$}

Ci permette di quantificare le sostanze azotate escrete con le urine. L'azoturia è determinante per valutare l'aderenza alle prescrizioni dietetiche, in quanto, con formule addette, è possibile risalire all'introito proteico (47): in una situazione di "steady-state", l'azoto escreto è uguale a quello introdotto (proteine introdotte $=($ Urea urinaria $+($ peso $*$ $\left.\left.\left.0 .{ }^{\prime} 25\right)\right) * 6.25\right)$.

\section{ALBUMINURIA E PROTEINURIA}

Fondamentale la sua valutazione nel paziente nefropatico, essendo espressione di danno renale, dovuto a un'alterata permeabilità dei capillari. L'albuminuria è considerata un

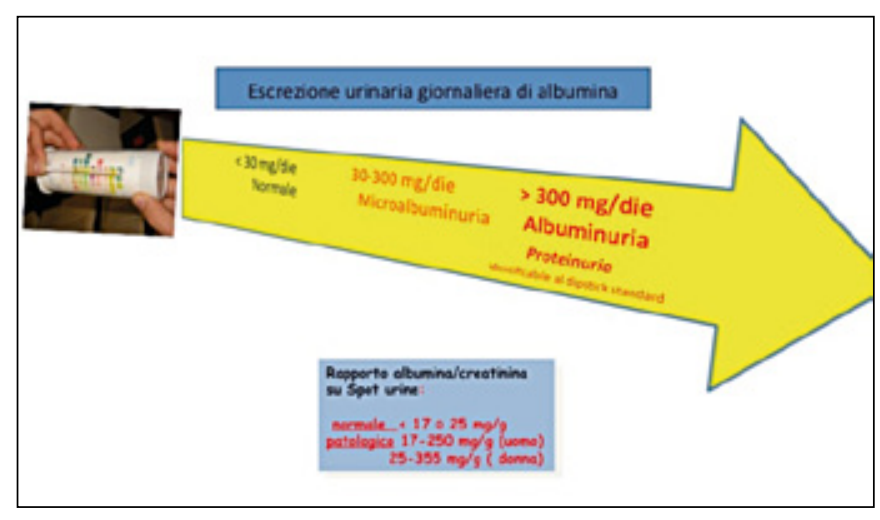

Fig 5 - Valori di microalbuminuria.

importante indicatore di rischio cardiovascolare, anche in soggetti senza un'importante riduzione della funzione renale (48). La proteinuria è definita come un'escrezione urinaria di proteine superiore a $150 \mathrm{mg}$ al giorno; altre classificazioni elevano tale soglia a $300 \mathrm{mg}$, tale per cui, sotto i $300 \mathrm{mg} /$ die, si parla di microalbuminuria e, sopra i 300 $\mathrm{mg} /$ die, si parla di proteinuria, intendendo con quest'ultimo termine, la perdita anche di altre proteine come immunoglobuline, transferrina e così via (49) (Fig. 5).

La determinazione di albuminuria e proteinuria è particolarmente importante nel follow-up del paziente, specialmente con nefropatia diabetica e cardiopatia, correlandosi con la progressione del danno renale. Il principale marker di danno renale nei pazienti diabetici e ipertesi è la microalbuminuria, che è definita come un aumento dell'escrezione urinaria di albumina.

È stato dimostrato, però, che l'aumento di microalbuminuria è un predittore di rischio $\mathrm{CV}$ anche nella popolazione generale.

La microalbuminuria è spesso associata ad altri danni d'organo nei pazienti ipertesi, come l'ipertrofia ventricolare sinistra, l'aterosclerosi carotidea e l'insulino-resistenza (50-52).

Nonostante ciò, è stato dimostrato che l'aumento dei valori di PA, in assenza di altri danni d'organo, può aumentare la filtrazione glomerulare di albumina, indipendentemente o meno dalla presenza di altre comorbidità; per tale ragione, viene ribadita l'importanza di tale marker urinario, in modo da poter quantificare il reale rischio CV. Molteplici studi di screening hanno avuto come oggetto la misurazione della microalbuminuria e del GFR, per individuare soggetti a rischio di sviluppare insufficienza renale cronica e complicanze CV. I dati hanno mostrato un significativo incremento del rischio di mortalità con valori elevati di microalbuminuria, indipendentemente dal GFR stimato (53). Un test semi-quantitativo, denominato dipstick urinario (basato sull'utilizzo di striscette reattive), viene utilizzato come esame di screening per la popolazione generale (54); qualora l'esito risulti positivo, il test può essere ripetuto a distanza di pochi giorni per escludere rialzi occasionali 
associati a condizioni non patologiche (p. es., attività fisica intensa, posizione ortostatica). Quando la diagnosi viene confermata o quando allo stick urinario si apprezzano proteinurie elevate, si procede alla raccolta delle urine nell'arco delle 24 ore; questa raccolta inizia solitamente la mattina, raccogliendo, ad ogni minzione, la diuresi dal secondo getto, includendo il primo della giornata successiva. Tale metodica risulta più precisa dal punto di vista quantitativo, ma spesso può essere inficiata da errori che possono alterare il risultato finale (p. es., incompleta raccolta del quantitativo urinario totale). Esistono, inoltre, diverse situazioni che possono falsare i valori di proteinuria e/o albuminuria, come presenza di infezioni delle vie urinarie, contaminazione da sangue, un esercizio fisico intenso e, anche, una prolungata stazione eretta (proteinuria "ortostatica") $24 \mathrm{~h}$ ore prima dell'esame.

$\mathrm{E}$, inoltre, possibile valutare le proporzioni e le concentrazioni delle varie proteine plasmatiche perse mediante elettroforesi urinaria (questo è particolarmente importante quando si sospetta che la proteinuria sia legata a un'aumentata sintesi di proteine plasmatiche, come succede nel mieloma multiplo).

Al posto del dipstick o del tradizionale esame delle urine nell' arco delle 24 ore, una maggiore accuratezza diagnostica è basata sul rapporto tra le concentrazioni urinarie di albumina e quelle di creatinina. Questo esame, noto anche come ACR, dall'inglese albumin-to-creatinine ratio, considera meritevole di approfondimenti diagnostici qualsiasi situazione in cui si registrino più di $30 \mathrm{mg}$ di albumina per ogni grammo di creatinina $(30 \mathrm{mg} / \mathrm{g}$ o 30 $\mathrm{mcg} / \mathrm{mg}$ ).

\begin{tabular}{|lll|}
\hline Categoria & $\begin{array}{l}\text { Rapporto } \\
\text { albuminuria/ } \\
\text { creatininuria } \\
\text { (mg/g) }\end{array}$ & $\begin{array}{l}\text { Albuminuria su raccolta } \\
\text { delle urine delle 24 ore } \\
\text { (mg/24 ore) }\end{array}$ \\
\hline Normale & Meno di 30 & Meno di 30 \\
\hline $\begin{array}{l}\text { Albuminuria } \\
\text { moderatamente aumentata }\end{array}$ & $30-300$ & $30-300$ \\
\hline $\begin{array}{l}\text { Albuminuria } \\
\text { significativamente } \\
\text { aumentata }\end{array}$ & Più di 300 & Più di 300 \\
\hline
\end{tabular}

Alcuni individui presentano un rischio più elevato di altri di sviluppare microalbuminuria, come, per esempio, pazienti diabetici, ipertesi, cardiopatici, con IRC (VFG $<60$ $\mathrm{mL} / \mathrm{min}$ ) o affetti da malattie sistemiche come lupus, artrite reumatoide o mieloma, per cui dovrebbero essere sottoposti con cadenza regolare al dosaggio dell'ACR.

\section{SODIURIA}

Ci permette di stimare l'introduzione di sodio con la dieta. La sodiuria in $\mathrm{mEq} /$ die diviso 17 ci fornisce i grammi di sale introdotti con la dieta.

Per tutti gli esami provenienti dalla raccolta urinaria ci sono alcune precauzioni, ma almeno due sono indispensabili (file PDF da scaricare e consegnare ai pazienti:
http://www.renalgate.it/perPazienti/raccolta urine.pdf). la modalità di raccolta delle urine sia come tempi che come quantità è fondamentale per evitare raccolte errate che possano inficiare i calcoli (è molto utile fornire uno schema ai pazienti in modo da istruirli su come eseguire correttamente la raccolta)

che il paziente sia in stato di equilibrio e che non abbia situazioni acute intercorrenti

Insieme allo screening biochimico e umorale, altre due misure sono fondamentali per l'assessment del paziente con IRC: la pressione arteriosa e il trattamento dietetico nutrizionale.

\section{PRESSIONE ARTERIOSA}

Più della metà dei pazienti affetti da nefropatia cronica (IRC) soffre di ipertensione arteriosa, che può contribuire all'evoluzione della nefropatia e che accresce il rischio di sviluppare malattie cardiache.

Seguire la terapia con attenzione e tenere sotto controllo la pressione arteriosa può aiutare a prevenire tali complicazioni.

La cura dell'ipertensione arteriosa in presenza di una nefropatia cronica prevede una dieta salutare, attività fisica e l'assunzione di farmaci specifici. La pressione arteriosa dovrebbe rimanere al di sotto della soglia dei 130/80 per i pazienti affetti da nefropatia.

Per pazienti diabetici affetti da nefropatia cronica o per pazienti con proteine nelle urine, $i$ farmaci più indicati contro l'ipertensione arteriosa sono gli inibitori dell'enzima di conversione dell'angiotensina (ACE) o i bloccanti del recettore per l'angiotensina (ARBS).

Il solo modo per sapere se la pressione arteriosa è troppo alta è misurarla. Normalmente, l'ipertensione è asintomatica; è per questo motivo che viene anche chiamata "killer silenzioso". Se dopo una sola misurazione i livelli di pressione risultano elevati, ciò non significa che si soffre di ipertensione; è necessario confermare tali valori in visite successive presso il proprio medico o il proprio ambulatorio.

Come si misura la pressione? Quanto spesso va controllata?

Di solito, per misurare la pressione, si usa un manicotto per la pressione, da posizionare intorno al braccio. È opportuno misurarla ogni volta che si effettua una visita dal proprio medico o presso il proprio ambulatorio, ma è anche possibile imparare a misurarsi la pressione da soli comodamente a casa. In questo modo, si possono controllare $\mathrm{i}$ valori quotidianamente e si può presentare al medico un resoconto dettagliato ogni volta che si effettua una visita. Per una corretta misurazione della pressione arteriosa, occorre rimanere seduti per alcuni minuti in un ambiente tranquillo, silenzioso e a temperatura confortevole.

Durante la misurazione, il bracciale deve trovarsi all'altezza del cuore. È preferibile e consigliabile misurare almeno tre volte la pressione arteriosa, con un intervallo tra le misurazioni di almeno mezzo minuto, con 2-3 minuti di distanza tra l'una e l'altra. 
Quando si controlla la pressione, vengono misurati due valori: il valore massimo corrisponde alla pressione sistolica, rilevata quando il cuore batte, e il valore minimo corrisponde alla pressione diastolica, rilevata nella pausa che il cuore fa tra un battito e l'altro. Un valore di $130 / 80$ è letto come $130 \mathrm{su} 80$. Valori normali di pressione negli adulti dai 18 anni in su sono inferiori a 120/80. In generale, valori di pressione pari a 140/90 o superiori sono considerati elevati.

L'Eighth Joint National Committee (JNC 8) ha alzato lievemente i valori normali: se prima si parlava, infatti, di $130 / 80$, ora si ha un'ipertensione considerata rischiosa sopra i $140 / 80$, sia nella popolazione normale che nei diabetici o negli ipertesi. Questo è un cambiamento epocale nel trattamento dell'ipertensione, che avrà sicuramente indubbie ricadute sia nella terapia che nella compliance dei pazienti (55).

\section{TRATTAMENTO DIETETICO NUTRIZIONALE (TDN)}

Il trattamento dietetico nutrizionale nell'IRC è in grado di aiutare a ridurre, fino ad annullare, molte delle complicanze che si sviluppano nella malattia renale. In particolare, il trattamento dietetico nutrizionale è in grado di ridurre il fosforo e, quindi, il $\mathrm{PTH}$, riduce l'acidosi, riduce il carico proteico che deve essere smaltito dai reni, riduce la malnutrizione, assicurando una quantità sufficiente di calorie e di aminoacidi ad alto valore biologico, e contribuisce a ridurre la potassiemia nel caso in cui questa si mostrasse elevata, magari per l'uso di ACEi o ARB.

In generale, i cardini del TDN sono: 1) la riduzione dell'introito dei cibi ad alto contenuto proteico (non solo carne, ma anche affettati, pesce, uova e formaggi, questi ultimi da non assumere, comunque, più di due volte alla settimana); 2) fornire calorie sufficienti favorendo una buona colazione, oltre a pasta e pane (quando il rischio di troppe proteine e troppo fosforo diventa alto per un'elevata assunzione, sono indicati i prodotti aproteici, in grado di disaccoppiare le calorie dal fosforo, dalle proteine e dal sodio); 3) ridurre l'introduzione di sodio a circa 5-6 grammi al giorno (attenzione al sodio nascosto nei cibi).

La trattazione esaustiva di tale trattamento è affidata ai suggerimenti sul trattamento dell'IRC pubblicati sul GIN (56). In generale, il TDN non è, quindi, solo in rapporto con il carico proteico, potendo ridurre la morte renale del $31 \%$, procrastinando la dialisi e riducendo $\mathrm{i}$ costi (57), ma è in grado di apportare numerosi effetti benefici nel metabolismo del malato renale.

\section{Riassunto}

L'insufficienza renale cronica (IRC) è una patologia in progressivo incremento; in Italia circa 3 milioni le persone affette da IRC agli stadi 3-5 sec. K-DOQI, ma una percentuale inferiore al $30 \%$ dei soggetti agli stadi $4-5$ sarebbe nota presso gli ambulatori nefrologici. A fronte di questa evidenza, che riflette importanti implicazioni nell'ambito della prevenzione primaria, si rilevano, il più delle volte, importanti difficoltà comunicative tra medico di medicina generale (MMG) e specialista nefrologo, in merito soprattutto alla tempistica e alle modalità di follow-up di questi pazienti. Alla luce di questo, abbiamo elaborato un Glossario che include informazioni riguardo all'approccio diagnostico e terapeutico delle complicanze dell'IRC, al fine di identificare precocemente le più frequenti anomalie metaboliche e di ottimizzare la tempistica dei controlli ambulatoriali.

Parole chiave: Insufficienza renale cronica, Paziente nefropatico, Follow-up, Complicanze

Dichiarazione di conflitto di interessi: Gli Autori dichiarano di non avere conflitto di interesse.

Contributi economici agli Autori: Gli Autori dichiarano di non aver ricevuto sponsorizzazioni economiche per la preparazione dell'articolo.

Indirizzo degli Autori:

Prof. Giuseppe Quintaliani

S.C. Nefrologia e Dialisi

Ospedale S.M. Misericordia

S. Andrea delle Fratte

06156 Perugia

quintaliani@yahoo.it

\section{Bibliografia}

1. Gambaro G, Yabarek T, Graziani MS, et al. Prevalence of CKD in northeasternItaly: results of the INCIPE study and comparison with NHANES. Clin J Am SocNephrol 2010; 5 (11): 1946-53.

2. An Estimated 26 Million Adults in the United States have Chronic Kidney Disease (CKD) http://www.cdc.gov/featu- res/dschronickidneydisease/.

3. Moranne O, Froissart M, Rossert J, et al. Timing of onset of CKD-related metabolic complications. J Am Soc Nephrol 2009; 20 (1): 164-71.

4. EDOTT Blog discussione perché i MMG devono essere sempre trascrittori nel gruppo Certificati online e Sanità elettronica.

5. http://www.mayoclinic.com/health/blood-urea-nitrogen/ 
MY00373. Elevation of BUN is predictive of long-term mortality in critically ill patients independent of "normal" creatinine.

6. Beier K, Eppanapally S, Bazick HS, et al. Elevation of BUN is predictive of long-term mortality in critically ill patients independent of "normal" creatinine. Crit Care Med 2011; 39 (2): 305-13.

7. Berg JM, Tymoczko JL, Stryer L. Biochemistry. 5th edition. New York: W H Freeman 2002.

8. Criego AB, Tkac I, Kumar A, Thomas W, Gruetter R, Seaquist ER. Brain glucose concentrations in healthy humans subjected to recurrent hypoglycemia. J Neurosci Res 2005; 82 (4): 525-30.

9. Comprehensive Clinical Clinical Nephrology. Chapter 66 Pathophysiology and etiology of acute kidney injury. Saunders, 2010, $4^{\circ}$ edizione.

10. Schena FP. Malattie dei reni e delle vie urinarie. Insufficienza renale acuta prerenale. McGraw-Hill Education, Giugno 2008: 398-9.

11. Guyton \& Hall. Fisiologia medica, i liquidi corporei e la funzione renale. Elsevier 2006: 326-7.

12. Froissart M, Rossert J, Jacquot C, Paillard M, Houillier P. Predictive performance of modification of diet in renal disease and Cockroft and Gault equations for estimating renal function. J Am Soc Nephrol 2005; 16 (3): 763-73.

13. http://www.sin-italy.org/Links/calcolatori_clinici.asp.

14. http://www.renalgate.it/programmi/clcr.xls.

15. Kidney Disease: Improving Global Outcomes (KDIGO) CKD-MBD Work Group. KDIGO clinical practice guideline for the diagnosis, evaluation, prevention, and treatment of Chronic Kidney Disease-Mineral and Bone Disorder (CKDMBD). Kidney Int Suppl 2009; 113: S1-130.

16. Patel RK, Oliver S, Mark PB, et al. Determinants of left ventricular mass and hypertrophy in hemodialysis patients assessed by cardiac magnetic resonance imaging. Clin J Am Soc Nephrol 2009; 4: 1477-83.

17. Foley RN, Curtis BM, Randell EW, Parfrey PS. Left ventricular hypertrophy in new hemodialysis patients without symptomatic cardiacdisease. Clin J Am Soc Nephrol 2010; 5: $805-13$.

18. Blacher J, Gueri AP, Pannier B, Marchais SJ, Safar ME, London GM. Impact of aortic stiffness on Survival in end-stage renal disease. Circulation 1999; 99: 2434-9.

19. Davies JE, Parker KJ, Francis DP, Hughes AD, Mayet J. What is the role of the aorta in directing coronary blood flow? Heart 2008; 94: 1545-7.

20. Arcidiacono T, Paloschi V, Rainone F, et al. Renal osteodystrophy and vascular calcification. J Endocrinol Invest 2009; 32 (4 Suppl.): 21-6.

21. Chue CD, Townend JN, Steeds RP, Ferro CJ. Arterial stiffnessin chronic kidney disease: causes and consequences. Heart 2010; 96: 817-23.

22. Floege J, Ketteler M. Vascular calcification inpatients withend-stage renal disease. Nephrol Dial Transplant 2004; 19 (Suppl. 5): V59-66.

23. Kovesdy CP, Ahmadzadeh S, Anderson JE, Kalantar-Zadeh $\mathrm{K}$. Secondary hyperparathyroidism is associated with higher mortality in men with moderate to severe chronic kidney disease. Kidney Int 2008; 73: 1296-302.
24. Tentori F, Blayney MJ, Albert JM, et al. Mortality risk fordialysis patients withdifferent levels of serum calcium, phosphorus, and PTH: The dialysis outcomes and practice patterns study (DOPPS). Am J Kidney Dis 2008; 52: 519-30.

25. Choi HS, Kim SH, Rhee Y, Cho MA, Lee EJ, Lim SK. Serum parathyroid hormone is associated with carotid intimamediathickness in postmenopausal women. Int J Clin Pract 2008; 62: 1352-7.

26. Hagstrom E, Ingelsson E, Sundstrom J, et al. Plasma parathyroidhormone and risk of congestive heart failure in the community. Eur J Hearth Fail 2010; 12: 1186-92.

27. Hedbäck G, Odén A. Increased risk of death from primary hyperparathyroidism--an update. Eur J Clin Invest 1998; 28: 271-6.

28. Lafferty F. Primary hyperparathyroidism. Changing clinical spectrum, prevalence of hypertension and discriminant analysis of laboratory tests. Arch Intern Med 1986; 141: 1761-6.

29. Neves KR, Graciolli FG, dos Reis LM, et al. Vascular calcification: contribution of parathyroid hormone in renal failure. Kidney Int 2007; 71: 1262-70.

30. Charbon GA. A rapid and selective vasodilator effect of parathyroid hormone. Eur J Pharmacol 1968; 3: 275-8.

31. Pang PK, Tenner TE Jr, Yee JA, Yang M, Janssen HF. Hypotensive action of parathyroid hormone preparations on rats and dogs. Proc Natl Acad Sci USA 1980; 77: 675-8.

32. Kawashima H. Parathyroid hormone causes a transient rise in intracellular ionized calcium in vascular smooth muscle cells. Biochem Biophys Res Commun 1990; 166: 709-14.

33. Oh J, Wunsch R, Turzer M, et al. Advanced Coronary and Carotid Arteriopathy in Young Adults With Childhood-Onset Chronic Renal Failure. Circulation 2002; 106: 100-5.

34. Coen G, Pierantozzi A, Spizzichino D, et al. Risk factors of one year increment of coronary calcifications and survival in hemodialysis patients. BMC Nephrol 2010; 21: 10-1.

35. Harnett JD, Parfrey PS, Griffiths SM, Gault MH, Barre P, Guttmann RD. Left ventricular hypertrophy in end-stage renal disease. Nephron 1988; 48: 107-15.

36. Liu X, Liu S. Rat parathyroid hormone 1-34 signals through the MEK/ERK pathway to induce cardiac hypertrophy. J Int Med Res 2008; 36: 942-50.

37. Amann K, Ritz E, Wiest G, Klaus G, Mall G. A role of parathyroid hormone for the activation of cardiac fibroblasts in uremia. J Am Soc Nephrol 1994; 4: 1814-9.

38. Cha H, Jeong HJ, Jang SP, et al. Parathyroid hormone accelerates decompensation following left ventricular hypertrophy. Exp Mol Med 2010; 42: 61-8.

39. Mall G, Huther W, Schneider J, Lundin P, Ritz E. Diffuse intermyocardiocytic fibrosis in uremic patients. Nephrol Dial Transplant 1990; 5: 39-44.

40. Saleh FN, Schirmer H, Sundsfjord CJ, Jorde R. Parathyroid hormone and left ventricular hypertrophy Eur Heart J 2003; 24: 2054-60.

41. Kestenbaum B, Sampson JN, Rudser KD, et al. Serum phosphate levels and mortality risk among people with chronic kidney disease. J Am Soc Nephrol 2005; 16: 520-8.

42. Tonelli M, Sacks F, Pfeffer M, Gao Z. Relation between serum phosphate level and cardiovascular event rate in people with coronary disease. Circulation 2005; 112: 2627-33. 
43. Jono S, McKee MD, Murry CE, et al. Phosphate regulation of vascular smooth muscle cell calcification. Circ Res 2000; 87: E10-7.

44. http://emedicine.medscape.com/article/242975-overview.

45. Chang CT, Chen YC, Fang JT, Huang CC. Metformin-associated lactic acidosis: case reports and literature review. J Nephrol 2002; 15 (4): 398-402.

46. Sharp Collaborative Group. Study of Heart and Renal Protection (SHARP): randomized trial to assess the effects of lowering low-density lipoprotein cholesterol among 9,438 patients with chronic kidney disease. Am Heart J 2010; 160 (5): 785-794.e10.

47. Maroni BJ, Steinman T, Mitch WE. A method for estimating nitrogen intake of patients with chronic renal failure. Kidney Int 1985; 27: 58-65.

48. Nicolas R, Robles, et al. Cross-sectional survey of the prevalence of reduced estimated glomerular filtration rate, albuminuria and cardiovascular risk in a native Spanish population. J Nephrol 2013; 26(4): 675-82.

49. Matsushita K, van der Velde M, Astor BC, et al. Association of estimated glomerular filtration rate and albuminuria with all-cause and cardiovascular mortality in general population cohorts: a collaborative meta-analysis.

50. Kohara K, Tabara Y, Tachibana R, Nakura J, Miki T. Microalbuminuria and arterial stiffness in a general Population: the Shimanami Health Promoting Program (J-SHIPP) Study. Hipertens Res 2204; 27 (7): 471-7.

51. Jager A, Kostense PJ, Ruhe HG, et al. Microalbuminuria and peripheral arterial disease are independent predictors of cardiovascular and all-cause mortality, especially among hypertensive subjects: five-year follow-up of the Hoorn
Study. Arterioscler Thromb Vasc Biol 1999; 19: 617-24.

52. Damsgaard EM, Froland A, Jorgensen OD, et al. Microalbuminuria as predictor of increased mortality in elderly people. BMJ 1990; 300: 297-300.

53. Hillege HL, Janssen WM, Bak AA, et al. Microalbuminuria is common, also in a nondiabetic, nonhypertensive population, and an independent indicator of cardiovascular risk factors and cardiovascular morbidity. J Intern Med 2001; 249 (6): 519-26.

54. Hillege HL, Fidler V, Diercks GF, et al. Urinary Albumin Excretion predicts Cardiovascular and Noncardiovascular Mortality in general population. Circulation 2002; 106 (14): 1777-82.

55. Yamagata K, Iseki K, Nitta K, et al. Chronic kidney disease perspectives in Japan and the importance of urinalysis screening. Clin Exp Nephrol 2008; 12: 1-8.

56. James PA, Oparil S, Carter BL, et al. 2014 Evidence-Based Guideline for the Management of High Blood Pressure in Adults: Report From the Panel Members Appointed to the Eighth Joint National Committee (JNC 8). JAMA 2014; 311 (5): 507-20.

57. Giuliano Brunori, et al. Terapia nutrizionale nell'IRC in fase conservativa: suggerimenti di pratica clinica e di applicazione delle Linee Guida. G Ital Nefrol 2013; 30 (Suppl. 59): 1-11.

58. Mennini FS, Russo S, Marcellusi A, Palazzo F, Quintaliani P. Cost-Effectiveness Analysis for the Treatment of Chronic Kidney Disease with Low-Protein Diet Functional Foods in Health and Disease. 2013; 3 (7): 291307. Page 291 of $306 \mathrm{http}: / /$ functionalfoodscenter.net/ files/72921645.pdf. 